\title{
Machine learning assisted quantification of graphitic surfaces exposure to defined environments
}

Cite as: Appl. Phys. Lett. 114, 241601 (2019); https://doi.org/10.1063/1.5095704

Submitted: 12 March 2019. Accepted: 06 May 2019 . Published Online: 17 June 2019

Chia-Yun Lai, Sergio Santos, and Matteo Chiesa

\section{COLLECTIONS}

F This paper was selected as Featured
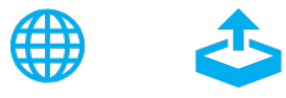

\section{ARTICLES YOU MAY BE INTERESTED IN}

Machine learning approach for analyzing complex data from atomic force microscopes

Scilight 2019, 250002 (2019); https://doi.org/10.1063/1.5114991

Determination of 3D electrostatic field at an electron nano-emitter

Applied Physics Letters 114, 013101 (2019); https://doi.org/10.1063/1.5055227

Quantum-enhanced noise radar

Applied Physics Letters 114, 112601 (2019); https://doi.org/10.1063/1.5085002

Lock-in Amplifiers Find out more today

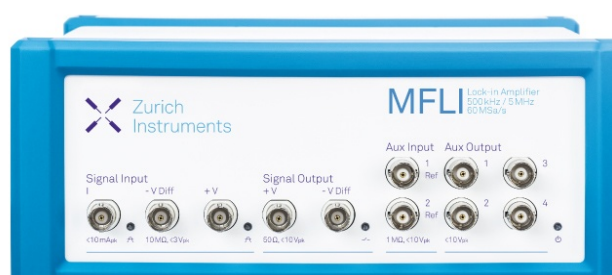

Zurich Instruments 


\title{
Machine learning assisted quantification of graphitic surfaces exposure to defined environments $\odot \rightarrow)$
}

\author{
Cite as: Appl. Phys. Lett. 114, 241601 (2019); doi: 10.1063/1.5095704 \\ Submitted: 12 March 2019 - Accepted: 6 May 2019 •
}

Published Online: 17 June 2019

Chia-Yun Lai, ${ }^{7}$ Sergio Santos, ${ }^{2}$ and Matteo Chiesa ${ }^{1,3, a)}$

\begin{abstract}
AFFILIATIONS
'Department of Physics and Technology, UiT The Artic University of Norway, 9037 Troms $\varnothing$, Norway

${ }^{2}$ Future Synthesis AS, Uniongata 18, 3732 Skien, Norway

${ }^{3}$ Laboratory for Energy and NanoScience (LENS), Khalifa University of Science and Technology, Masdar Institute Campus, 127788 Abu Dhabi, United Arab Emirates
\end{abstract}

${ }^{a)}$ Author to whom correspondence should be addressed: matteo.chiesa@uit.no

\begin{abstract}
We show that it is possible to submit the data obtained from physical phenomena as complex as the tip-surface interaction in atomic force microscopy to a specific question of interest and obtain the answer irrespective of the complexity or unknown factors underlying the phenomena. We showcase the power of the method by asking "how many hours has this graphite surface been exposed to ambient conditions?" In order to respond to this question and with the understanding that we have access to as many experimental data points as needed, we proceed to label the experimental data and produce a "library." Then, we submit new data points to the test and request the model contained in this library answers to the question. We show that even with a standard artificial neural network, we obtain enough resolution to distinguish between surfaces exposed for less than $1 \mathrm{~h}$, up to $6 \mathrm{~h}$, and $24 \mathrm{~h}$. This methodology has potential to be extended to any number of questions of interest.
\end{abstract}

Published under license by AIP Publishing. https://doi.org/10.1063/1.5095704

There is arguably a divide in the approach to finding answers to questions in the general sciences. The first one has two parts and consists in either looking into phenomena and finding expressions corresponding to fundamental laws, i.e., the strictest theoretical side of the sciences, or explaining phenomena probed experimentally directly from these first principles as mechanisms that provide the physical insight into our findings, i.e., the practical science that exploits the link between experiment and the strictest theory. The second consists in asking a question to a phenomenon and finding a correlation, i.e., an association in general, between knowns, i.e., the data that we have access to, and the unknowns, i.e., the answer to the question we are asking. We could argue that the task of the second method to finding answers to questions was typically dealt with by statistics for a long time.

In recent years, computer systems have considerably enabled and extended the second form of enquiry by allowing computing over extremely large sets of data, i.e., big data, rapidly and efficiently, i.e., via artificial neural networks (ANNs), vector supporting machines, etc., and, importantly, via the exploitation of these "model free" algorithms that build a model without being explicitly programed to, in order to answer a specific question based on data alone. ${ }^{1}$ Arguably, the last point defines the field of machine learning (ML). It is now possible to find standard tool sets in any standard programming language, i.e., Matlab, Python, or R, which include powerful ML algorithms to solve problems in this way. ${ }^{2}$ It is not clear, however, where the two methods that we pointed out earlier meet or will meet, but we claim that it is at least advantageous to be able to answer specific questions of practical relevance from sets of data extracted from complex physical systems for which fundamental laws, principles, or expressions are either too complex or unavailable. ${ }^{3}$

In the field of atomic force microscopy (AFM), a sharp tip of nanometric radius held at the end of a microscopic cantilever is made to interact with a surface [Fig. 1(a)]. In the interaction, complex conservative and dissipative phenomena ${ }^{4}$ affect the dynamics of the cantilever. ${ }^{5}$ A main paradigm of the field has been to extract information from the dynamics in relation to the properties of the surfaces. ${ }^{6,7}$ On the other hand, the AFM field expands via two main fronts. Namely, (1) improving the instrumentation so the data itself contain more 
(a)

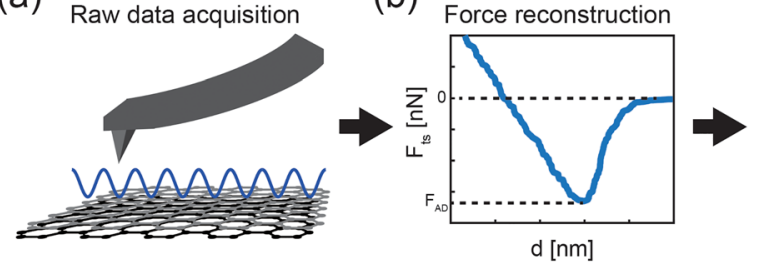

(c)

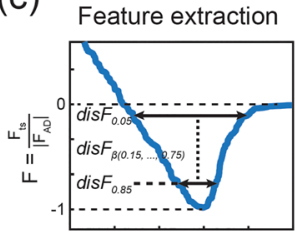

$\mathrm{d}[\mathrm{nm}]$ (b)

(d) $\mathrm{d}[\mathrm{nm}]$

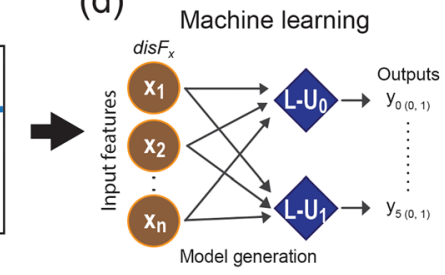

FIG. 1. Illustrations of the process from (a) acquiring experimental raw data, (b) preparing experimental AFM force data, (c) schematics of discrete $x$ input (distance in black arrows) construction from force data, and (d) schematics of the generation of ANN models.

information of this tip-surface interaction ${ }^{8,9}$ and (2) improving the data processing, modeling, and overall understanding of the phenomena in order to understand and interpret the available data. ${ }^{10}$ It is not clear whether more information can be extracted from the phenomena, that is, whether enhancing the resolution of the system would provide more information about the nanoscale properties of the surface or whether we have reached a point where data processing is the main bottleneck in terms of advancing in the field. ${ }^{11,12}$ On the other hand, we have recently shown that simple power laws ${ }^{13}$ are not enough to explain the rich phenomena that we have already probed in ambient conditions, where air contaminants, such as $\mathrm{CO}_{2}$ and water, start adhering to the surface almost immediately after surfaces are produced. ${ }^{14}$ This surface phenomenon under ambient conditions, which we term here "surface aging," is a dynamic process with many unknowns and possibly related to very complex physical processes such as ion exchange, surface energy dynamics, and surface-tension thermodynamic equilibrium, ${ }^{15,16}$ thus providing us with a suitable candidate question in AFM. Namely, "how long has a surface been exposed to ambient conditions?" In principle, we expect that the tipsurface force will change with time and that these changes can be associated with our question via ML algorithms, provided real phenomena underlying surface aging truly affect the surface.

We employ Highly Ordered Pyrolytic Graphite (HOPG) as a model sample because it is easy to cleave with standard scotch tape. In this way, HOPG is cleaved creating a pristine surface and labeled $0 \mathrm{~h}$. After cleaving, the sample is exposed to ambient air [temperature $\mathrm{T}$ $\approx 22 \pm 2{ }^{\circ} \mathrm{C}$ and relative humidity $\left.(\mathrm{RH}) \approx 50 \% \pm 5 \%\right]$ for $24 \mathrm{~h}$ at which point, and in principle, phenomena, known or unknown by us as researchers, will act on the surface inducing the surface aging phenomenon. In any case, we stand by our simple question "how old is this surface in hours?" and attend simply to the outcome of the ML algorithm.

Here, we used an ANN as the chosen supervised ML method to generate our predictive model. A set of discrete inputs x, i.e., the knowns, must be paired to a set of discrete outputs $y$ which are the

predictions, i.e., our set of example pairs $(\mathrm{x}, \mathrm{y})$. The $\mathrm{y}$ values are to be later predicted by the model, while initially these are known in order to generate an optimized model from the set of (training) pairs and hence the name supervised. ${ }^{17}$ Finding such an optimized model is the "job" of the ANN while ours consists in initially discretizing AFM data [blue lines in Figs. 1(b) and 1(c)] and converting them to valid (discrete) inputs $\mathrm{x}$ mapping to $\mathrm{y}$. A complete set of experiments for us consists of data taken in 6-time steps: $0 \mathrm{~h}+0.2 \mathrm{~h}, 1 \mathrm{~h} \pm 0.2 \mathrm{~h}$, $3 \mathrm{~h} \pm 0.2 \mathrm{~h}, 6 \mathrm{~h} \pm 0.2 \mathrm{~h}, 12 \mathrm{~h} \pm 0.2 \mathrm{~h}$, and $24 \mathrm{~h} \pm 0.2 \mathrm{~h}$. The error in time is due to the time (approx. $15 \mathrm{~min}$ ) it takes to take the 600 data points (data point identified as the force curve) for each time interval. The 6 time slots provide the 6 -tuple output $\mathrm{y}=\left\{\mathrm{y}_{0}, \ldots, \mathrm{y}_{5}\right\}$ [Fig. 1(d)] for our model where each $y_{i}$ belongs to a given time slot. Each $n$-tuple input $\mathrm{x}=\left\{\mathrm{x}_{0}, \ldots, \mathrm{x}_{\mathrm{n}}\right\}$ taken during a given time slot $\mathrm{y}_{\mathrm{i}}$ is thus paired. The idea is simple. For example, if we feed AFM data ( $\mathrm{x}$ ) taken during the time interval corresponding to $y_{0}$ to the model, the output should be $\mathrm{y}_{0}=1$ and all other $\mathrm{y}_{\mathrm{i}}=0$. When this happens, we have a True Positive (TP). If $\mathrm{y}_{0}=0$, we have a False Negative (FN). True Negatives (TN) and False Positives (FP) are similarly defined. As a criterion to optimize our ANN, we used the $\mathrm{F}_{1}$ score, which is based on maximizing $\mathrm{TP}$ and TN while minimizing FP and FN and is a standard in ML. ${ }^{18}$

Having defined the y (output) data, our task now consists in defining how to convert AFM data into (discrete) x data. Our AFM data consist of force-distance $\left(F_{\text {ts }}\right.$ vs $\left.d\right)$ curves or force data, as shown in blue lines in Figs. 1(b) and 1(c). ${ }^{4-6}$ Since not even such force data are obtainable directly in AFM, however, here we employ standard dynamic AFM to first acquire data and then convert them into force data. ${ }^{6,7}$ The cantilever oscillates with free amplitude $\mathrm{A}_{0}$ at the free resonance and it allows reconstructing the $\mathrm{F}_{\mathrm{ts}}$ vs $\mathrm{d}$ curves directly from 2 experimental observables, i.e., the change in amplitude A and phase $\Phi$. In short, (1) monitoring $A$ and $\Phi$ while approaching the surface gives us experimental Amplitude (A) and Phase ( $\Phi$ ) Distance (APD) curves; (2) from the APDs, we exploit the Sader-Jarvis-Katan formalism ${ }^{19,20}$ for the conversion into $F_{t s}$ vs d curves (Fig. 1(b); and (3) finally, we transform $\mathrm{F}_{\mathrm{ts}}$ vs $\mathrm{d}$ to discrete $\mathrm{x}$ input data [distances as black arrows in Fig. 1(c)]. For steps 1 and 2, the data were collected with a Cipher AFM (Asylum Research-Oxford Instruments) and standard OLYMPUS AC160TS cantilevers with $\mathrm{k}$ (spring constant) $\approx 30 \mathrm{~N} / \mathrm{m}$, $\mathrm{f}_{0}$ (natural frequency) $\approx 300 \mathrm{kHz}$, and $\mathrm{Q} \approx 400$; standard thermal analysis provides the $\mathrm{f}_{0}$ and $\mathrm{Q}$ values. ${ }^{21} \mathrm{~F}_{\mathrm{ts}}$ vs $\mathrm{d}$ was obtained by numerically integrating the expression

$$
\mathrm{F}_{t s}(\mathrm{~d})=2 \mathrm{k} \int_{u=d}^{u=\infty}\left[\left(1+\frac{A^{1 / 2}(u)}{8 \sqrt{\pi(u-d)}}\right) \Omega(u)-\frac{A^{3 / 2}(u)}{\sqrt{2(u-d)}} \frac{d \Omega(u)}{d u}\right] d u,
$$

where $\Omega$ is the normalized frequency shift,

$$
\Omega(\mathrm{d})=\left[1+\frac{A_{0}}{Q A} \cos (\Phi(d))\right]^{\frac{1}{2}}-1 .
$$

Further details can be found elsewhere. ${ }^{22,23}$ Since it is well known that the tip radius $\mathrm{R}$ strongly affects the tip-sample interaction, the effective tip radius $\mathrm{R}$ was monitored before and after each experiment with the use of the critical amplitude $A_{C}$ method; ${ }^{24} R=8 \mathrm{~nm} \pm 2 \mathrm{~nm}$ throughout as expected according to the OLYMPUS manufacturer. This method has the advantage of being in situ and fast, i.e., it takes seconds 
TABLE I. Experimental normalized disF values: example of 2 sets of input features disF for each time step used to generate models for HOPG aging under ambient conditions. The first column stands for the $\beta$ parameter and the other columns for the normalized disF values at each time period. Set 1 consists of the values generated by averaging the first 50 AFM data points and set 2 by the data points from 51 to 100 (for all sets, see the supplementary material).

\begin{tabular}{lcccccccccccc}
\hline \hline$\beta$ & 0 h set 1 & 0 h set 2 & 1 h set 1 & 1 h set 2 & 3 h set 1 & 3 h set 2 & 6 h set 1 & 6 h set 2 & 12 h set 1 & 12 h set 2 & 24 h set 1 & 24 h set 2 \\
\hline 0.75 & 0.91 & 0.94 & 0.92 & 0.87 & 0.93 & 0.92 & 0.92 & 0.93 & 0.93 & 0.95 & 0.93 & 0.92 \\
0.65 & 0.83 & 0.84 & 0.85 & 0.81 & 0.88 & 0.87 & 0.85 & 0.85 & 0.88 & 0.89 & 0.85 & 0.84 \\
0.55 & 0.75 & 0.77 & 0.79 & 0.75 & 0.82 & 0.81 & 0.79 & 0.78 & 0.83 & 0.83 & 0.76 & 0.76 \\
0.45 & 0.67 & 0.69 & 0.68 & 0.68 & 0.74 & 0.73 & 0.72 & 0.72 & 0.76 & 0.76 & 0.67 & 0.66 \\
0.35 & 0.57 & 0.60 & 0.61 & 0.57 & 0.63 & 0.63 & 0.65 & 0.65 & 0.67 & 0.67 & 0.57 & 0.57 \\
0.25 & 0.44 & 0.47 & 0.50 & 0.48 & 0.50 & 0.47 & 0.55 & 0.55 & 0.53 & 0.54 & 0.46 & 0.48 \\
0.15 & 0.30 & 0.31 & 0.34 & 0.32 & 0.29 & 0.29 & 0.39 & 0.38 & 0.33 & 0.37 & 0.32 & 0.32 \\
0.05 & 0.12 & 0.12 & 0.13 & 0.12 & 0.11 & 0.12 & 0.15 & 0.15 & 0.15 & 0.13 & 0.14 & 0.13 \\
\hline \hline
\end{tabular}

to monitor R. Only tips for which $\mathrm{R}$ was found to fall into this range were used. Furthermore, we used cantilevers from a single cantilever wafer. Since the wafer had been fabricated at least months prior to its use and given that our experiments were conducted for a few consecutive days only, we considered the effects of this type of cantilever aging negligible. In the future, however, $\mathrm{R}$ could be included as an input feature in $\mathrm{x}$.

After acquiring the $\mathrm{F}_{\mathrm{ts}}$ vs $\mathrm{d}$ data [Fig. 1(b)], these profiles were parameterized to generate standardized input features $\mathrm{x}$ [Figs. 1(c) and 1(d)] to answer our specific question. The method to generate the $x$ features was somehow arbitrary and consisted in parametrizing and normalizing the distances in the tip-sample well in the $\mathrm{F}_{\mathrm{ts}}$ vs $\mathrm{d}$ curves [see black arrows in Fig. 1(c)]. The well contains information from both attractive and repulsive interactions. ${ }^{5}$ The procedure is as follows: (1) minima were identified with the force of adhesion $\mathrm{F}_{\mathrm{AD}}$ and its absolute value was used to normalize $\mathrm{F}_{\mathrm{ts}}$ as $\mathrm{F}=\mathrm{F}_{\mathrm{ts}} /\left|\mathrm{F}_{\mathrm{AD}}\right|[\mathrm{Fig} .1(\mathrm{c})]^{25}$ (2) A factor $\beta$ was introduced to parametrize the well for $\mathrm{F}<0$ (net attractive); by varying $\beta$ from 0 to 1 , where $-\mathrm{F}=\beta, \mathrm{F}$ is parametrized. (3) A set of $\beta$ values was chosen to represent the well. An ANN might not significantly increase its predictive power by increasing the number of input features. Furthermore, computational power is compromised by indiscriminately increasing this number. By inspection, we find that taking $\beta=0.05,0.15, \ldots, 0.85$, i.e., a total of 9 (minus 1 ) input features $\mathrm{x}$ worked well. (4) We measured the distances in $\mathrm{nm}$ that "cut" $\mathrm{F}$ in the horizontal line, thus producing a distance for each $\beta$, i.e., $\operatorname{disF}_{\beta}$ [see black arrows in Fig. 1(c)]. These are the discrete features $\mathrm{x}$ in $\mathrm{nm}$ before normalizing. (5) We then normalized by computing $\operatorname{disF}_{\mathrm{i}}=\operatorname{disF}_{\beta} / \operatorname{disF}_{0.85} ; \mathrm{i}=1$ to 8 (where $\mathrm{x}$ is finally identified with $\operatorname{disF}_{\mathrm{i}}$ as an 8-tuple for each curve). (6) Finally, in order to reduce random error, the final dis $\mathrm{F}_{\mathrm{i}}$ were computed by averaging for every $\approx 50 \mathrm{AFM}$ data points for each time interval or step. These $\operatorname{dis}_{\mathrm{i}}(\mathrm{x})$ features [Fig. 1(d)] were fed into our ANN. In Table I, we provide numerical values of $\operatorname{dis}_{\mathrm{i}}$ for the first two input datasets that were acquired for each time step (see the supplementary material for the numerical values of the complete list of sets). The data were acquired with many different tips, then randomized, and finally averaged. Each set corresponds to approximately 50 data points taken for these random sets (taken with several cantilevers with only constant being the time slot). A total of 56 sets similar to those shown in Table I (see the supplementary material) were employed to generate a library to monitor the HOPG aging process, i.e., an HOPG aging library; this library is simply our final optimized predictive model produced by our ANN, ${ }^{7,13}$ and the selection and performance of the model library are discussed below. Figure 2 shows some of the force data and an

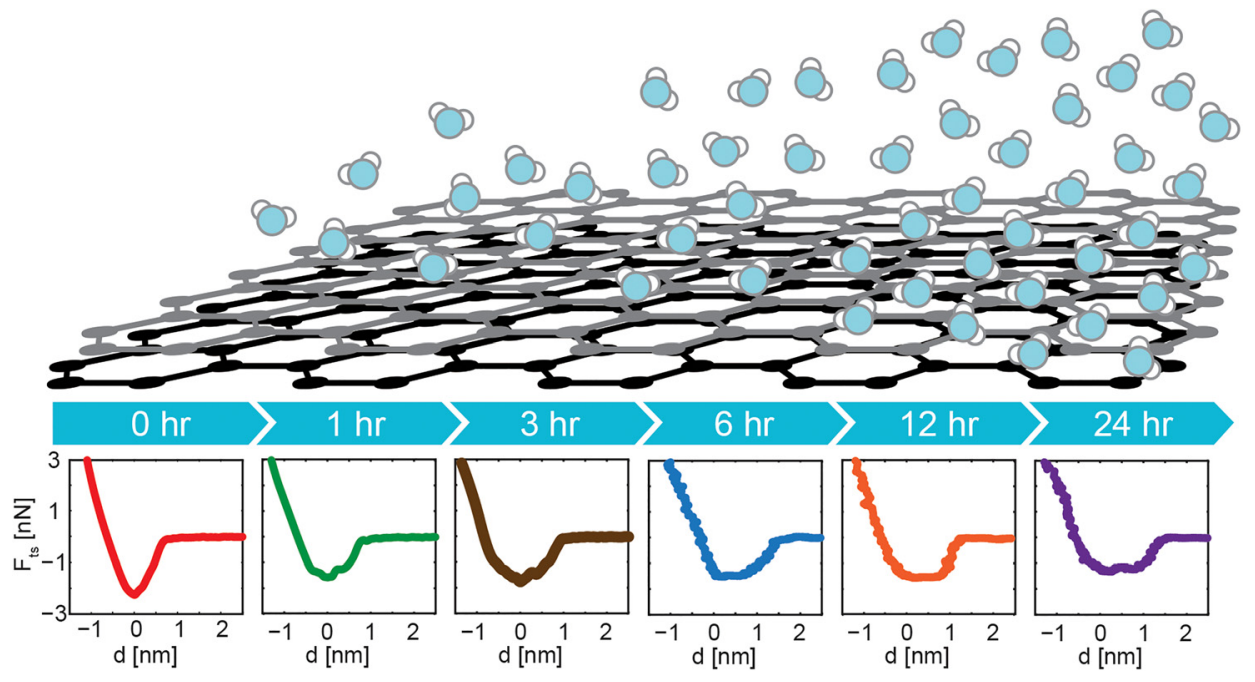

FIG. 2. Evolution of force $F_{t s} v s d$ profiles at each time step in hours $h$, as the HOPG surface ages by exposure to ambient conditions. 
illustration of the changes on the surface that might have produced the difference in the AFM "signal." It is worth nothing that while we represent water-adhesion in the illustration, knowledge of this physical phenomenon is, in principle, not required in order to answer our question.

Our ANN was implemented in Matlab as explained in Ref. 7. Our implementation of the ANN also considered optimizing the number of $\mathrm{L}$ layers and $\mathrm{U}$ units per layer in the network by exploiting the $\mathrm{F}_{1}$ score criterion described above throughout. First, we submitted training sets consisting of $(\mathrm{x}, \mathrm{y})$ pairs to the ANN and models were produced. To optimize these models, we cross-validated them as it is the standard procedure in ML. This consists in submitting the models produced by the training sets to a test, i.e., cross-validation, where now the models do not "know" the true output y. Supervision and error monitoring are thus possible since we do know y (supervised training) and the best models, i.e., producing higher $F_{1}$ score, from the training set are selected according to the $\mathrm{F}_{1}$ score given by cross-validation. An example of the $\mathrm{F}_{1}$ score results is given in Table II. We also provide the Precision and Recall values for each model since these provide further information about errors, i.e., $\mathrm{FP}$ and $\mathrm{FN}$. $^{18}$

We took the model with the highest $\mathrm{F}_{1} \approx 0.78$ score (Table II, $3 \mathrm{~L}$ and $3 \mathrm{U}$ ) and tested it with data the model had never seen before (test sets). For this, a different tip was used, and data were collected as before. Then, we tested the 3L-3U model against these testing sets. Since $y=1$ is hardly the case practically and since $y$ ranges from 0 to 1 instead [see Fig. 1(d)], we considered y $>0.8$ as 1 and the rest 0 , i.e., $y>0.8$ was selected as the threshold for positive identification or $y_{i}$ $=1$. Such thresholds are standard in ANN implementations and are typically selected by inspection. The outcome of the predictions is shown in Fig. 3. The open circles stand for either FP or FN, i.e., incorrect predictions, while the solid circles indicate $\mathrm{TP}$ or TN, i.e., correct predictions. By testing with data collected at $1 \mathrm{~h}$ of surface aging (light blue background), the model produced only 7 FPs (out of 16 data points) with most of the errors falling into the $0 \mathrm{~h}$ range [Fig. 3(a)]. On the other hand, there were $10 \mathrm{TP}$ and $6 \mathrm{FN}$ for the $1 \mathrm{~h}$ set. In Figs. 3(b) and $3(\mathrm{c})$, we show the predictions obtained by submitting data from $6 \mathrm{~h}$ and $24 \mathrm{~h}$ test sets to the generic model, respectively. The results are 5 and 1 FPs for the $6 \mathrm{~h}$ ( 8 FNs and 9 TPs) and $24 \mathrm{~h}$ ( 3 FNs and 13 TPs) datasets, respectively (out of 17 data points per set).

In summary, we have shown that a standard Neural Network can be employed to predict the time of exposure to ambient conditions of a graphite surface directly from AFM data. No knowledge of the underlying phenomena is required in order to predict the time of

TABLE II. The performance of $2 \mathrm{~L} 2 \mathrm{U}, 2 \mathrm{~L} 3 \mathrm{U}, 3 \mathrm{~L} 2 \mathrm{U}, 3 \mathrm{~L} 3 \mathrm{U}, 4 \mathrm{~L} 2 \mathrm{U}$, and $4 \mathrm{~L} 4 \mathrm{U}$ models is evaluated by the $F_{1}$ score criterion. These models were generated with the input features shown in the supplementary material and then tested with separate data from different time steps (cross-validation).

\begin{tabular}{lccc}
\hline \hline & Precision & Recall & $\mathrm{F}_{1}$ score \\
\hline 2L 2U & 0.79 & 0.63 & 0.70 \\
2L 3U & 0.79 & 0.63 & 0.70 \\
3L 2U & 0.93 & 0.58 & 0.72 \\
3L 3U & 0.82 & 0.75 & 0.78 \\
4L 2U & 1.00 & 0.00 & 0.00 \\
4L 3U & 0.75 & 0.75 & 0.75 \\
\hline \hline
\end{tabular}
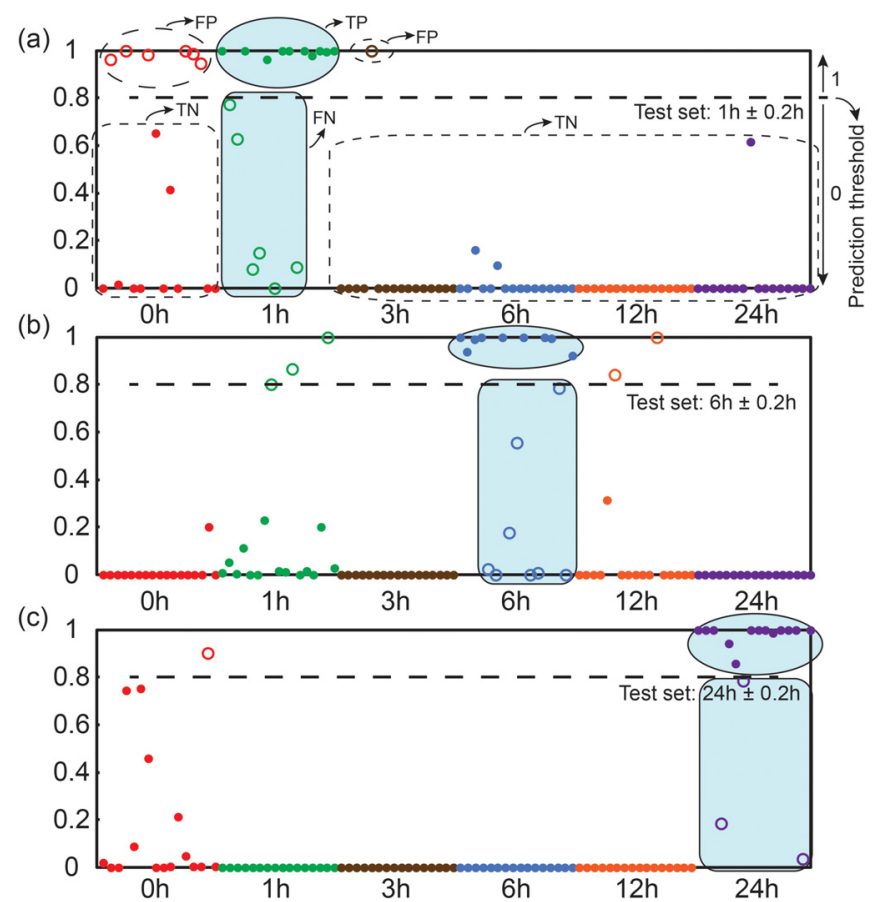

FIG. 3. Predictions of the $3 \mathrm{~L} 3 \mathrm{U}$ model against input datasets at $1 \mathrm{~h}(\mathrm{a}), 6 \mathrm{~h}(\mathrm{~b})$, and $24 \mathrm{~h}$ (c). The open circles stand for FN or FP, while the solid circles indicate TP or TN. Color codes are used to refer to the specific time steps: $0 \mathrm{~h}$ (red), $1 \mathrm{~h}$ (green), $3 \mathrm{~h}$ (brown), $6 \mathrm{~h}$ (blue), $12 \mathrm{~h}$ (orange), and $24 \mathrm{~h}$ (purple). The light blue regions indicate the only output $y_{i}$ that should be 1 in each case.

exposure. The complexity of the data and difficulty to reduce them to well-known first principles might in fact act as a positive factor in terms of enhancing the predictive power of the model since ANN and other ML methods benefit from complexity and details. We propose that model libraries can be employed to predict phenomena that answer specific questions in AFM and that libraries could be customized and standardized in a similar way to that presented here.

See the supplementary material for complete input features that are used to train ANN and generate models.

C. Y. Lay and M.C. acknowledge the support of the Arctic Center for Sustainable Energy (ARC), UiT Arctic University of Norway through Grant No. 310059.

\section{REFERENCES}

${ }^{1}$ L. Bottou, F. E. Curtis, and J. Nocedal, SIAMR 60(2), 223 (2018).

${ }^{2}$ F. Pedregosa, G. Varoquaux, A. Gramfort, V. Michel, B. Thirion, O. Grisel, M. Blondel, P. Prettenhofer, R. Weiss, and V. Dubourg, J. Mach. Learn. Res. 12, 2825 (2011).

${ }^{3}$ N. Borodinov, S. Neumayer, S. V. Kalinin, O. S. Ovchinnikova, R. K. Vasudevan, and S. Jesse, Comput. Mater. 5(1), 25 (2019).

${ }^{4}$ J. P. Cleveland, B. Anczykowski, A. E. Schmid, and V. B. Elings, Appl. Phys. Lett. 72(20), 2613 (1998).

5. Tamayo and R. Garcia, Appl. Phys. Lett. 73(20), 2926 (1998).

${ }^{6}$ S. Santos, C.-Y. Lai, T. Olukan, and M. Chiesa, Nanoscale 9(16), 5038 (2017).

${ }^{7}$ S. Santos, C.-Y. Lai, C. A. Amadei, K. R. Gadelrab, T.-C. Tang, A. Verdaguer, V. Barcons, J. Font, J. Colchero, and M. Chiesa, Nanoscale 8(40), 17400 (2016). 
${ }^{8}$ S. Jesse, R. K. Vasudevan, L. Collins, E. Strelcov, M. B. Okatan, A. Belianinov, A. P. Baddorf, R. Proksch, and S. V. Kalinin, Annu. Rev. Phys. Chem. 65(1), 519 (2014).

${ }^{9}$ O. Sahin, S. Magonov, C. Su, C. F. Quate, and O. Solgaard, Nat. Nanotechnol. 2, 507 (2007).

${ }^{10}$ R. Garcia and E. T. Herruzo, Nat Nanotechnol. 7(4), 217 (2012).

${ }^{11}$ S. Benaglia, V. G. Gisbert, A. P. Perrino, C. A. Amo, and R. Garcia, Nat. Protoc. 13(12), 2890 (2018).

${ }^{12}$ Y. M. Efremov, A. X. Cartagena-Rivera, A. I. M. Athamneh, D. M. Suter, and A. Raman, Nat. Protoc. 13(10), 2200 (2018).

${ }^{13}$ C.-Y. Lai, T. Olukan, S. Santos, A. Al Ghaferi, and M. Chiesa, Chem. Commun. 51(99), 17619 (2015).

${ }^{14} \mathrm{~S}$. Santos and A. Verdaguer, Materials 9(3), 182 (2016).

${ }^{15}$ T. E. Balmer, H. K. Christenson, N. D. Spencer, and M. Heuberger, Langmuir 24(4), 1566 (2007).
${ }^{16}$ H. K. Christenson and N. H. Thomson, Surf. Sci. Rep. 71(2), 367 (2016).

${ }^{17}$ S. Raschka and V. Mirjalili, Python Machine Learning (Packt Publishing Ltd., 2017).

${ }^{18}$ D. M. Powers, J. Mach. Learn. Technol. 2, 37 (2011).

${ }^{19}$ J. E. Sader and S. P. Jarvis, Appl. Phys. Lett. 84(10), 1801 (2004).

${ }^{20}$ A. J. Katan, M. H. van Es, and T. H. Oosterkamp, Nanotechnology 20(16), 165703 (2009).

${ }^{21}$ J. L. Hutter and J. Bechhoefer, Rev. Sci. Instrum. 64(7), 1868 (1993).

${ }^{22}$ C. A. Amadei, S. Santos, S. O. Pehkonen, A. Verdaguer, and M. Chiesa, J. Phys. Chem. C 117(40), 20819 (2013).

${ }^{23}$ S. Santos, C. A. Amadei, T. C. Tang, V. Barcons, and M. Chiesa, preprint arXiv:1401.6587 (2014).

${ }^{24}$ S. Santos, L. Guang, T. Souier, K. Gadelrab, M. Chiesa, and N. H. Thomson, Rev. Sci. Instrum. 83(4), 043707 (2012).

${ }^{25}$ R. Garcia and A. S. Paulo, Phys. Rev. B 60(7), 4961 (1999). 